TRANSACTIONS OF THE

AMERICAN MATHEMATICAL SOCIETY

Volume 326, Number 2, August 1991

\title{
PLANE AUTONOMOUS SYSTEMS WITH RATIONAL VECTOR FIELDS ${ }^{1}$
}

\author{
HAROLD E. BENZINGER
}

\begin{abstract}
The differential equation $\dot{z}=R(z)$ is studied, where $R$ is an arbitrary rational function. It is shown that the Riemann sphere is decomposed into finitely many open sets, on each of which the flow is analytic and, in each time direction, there is common long-term behavior. The boundaries of the open sets consist of those points for which the flow fails to be analytic in at least one time direction. The main idea is to express the differential equation as a continuous Newton method $\dot{z}=-f(z) / f^{\prime}(z)$, where $f$ is an analytic function which can have branch points and essential singularities. A method is also given for the computer generation of phase plane portraits which shows the correct time parametrization and which is noniterative, thereby avoiding the problems associated with the iteration of rational functions.
\end{abstract}

\section{INTRODUCTION}

This paper gives a geometric description of the phase plane portraits (in the extended complex plane) of the differential equation

$$
\dot{z}=R(z),
$$

where $R$ is any rational function of the complex parameter $z, \dot{z}$ means $d z / d t$, and $t$ is a real-valued independent variable. The main tool in this work is the representation of $R$ as

$$
R(z)=-f(z) / f^{\prime}(z)
$$

where $f$ is a (generally multivalued) analytic function, unique up to a multiplicative constant (see $\S 2$ ). Thus (1.1) takes the form

$$
\dot{z}=-f(z) / f^{\prime}(z) \text {, }
$$

the continuous Newton method of $f$, and $R$ is the continuous Newton map of $f$.

Received by the editors August 9, 1988 and, in revised form, March 1, 1989.

1980 Mathematics Subject Classification (1985 Revision). Primary 34A20, 34A34, 34C35, $65 \mathrm{~L} 99,65 \mathrm{~S} 05$.

Key words and phrases. Phase plane portraits, analytic flows, continuous Newton method, computer graphics.

1 This paper is being published posthumously. Offprints of the article may be obtained from Dr. Lee A. Benzinger, Loral WDL, P. O. Box 49041, M/S X-20, San Jose, California 95161-9041 (E-mail address: LAB@WDL1.WDL.LORAL.COM). 
The main results are:

1. The extended plane is decomposed into a finite number of open sets $U$, each invariant under the flow defined by (1.3), and the flow is analytic on $U$. Either all trajectories in $U$ are periodic, or all of them converge in forward and backward time to equilibrium solutions of (1.3). In particular, there are no limit cycles.

2. The complement in the extended plane of the open sets $U$ consists of the finitely many trajectories of (1.3) for which the flow is not analytic in at least one direction.

3. The local behavior of trajectories in a neighborhood of a zero of $R$ is determined by the leading term of $R$, even if the zero is of multiplicity greater than one.

4. The correct time parametrization of (1.1) can be obtained from the orthogonal trajectories arising from

$$
\dot{z}=i R(z) \text {. }
$$

The geometric description of phase plane portraits was initiated by Poincaré [8] for autonomous systems

$$
d x / X=d y / Y \text {, }
$$

where $X$ and $Y$ are polynomials in $x$ and $y$. Equation (1.1) is a special case of (1.5), if one is willing to give up information on the parametrization of the trajectories. If $R(z)=-p(z) / q(z)$, where $p, q$ are polynomials with no common factors, then $R(z)=-p(z) \bar{q}(z) /|q(z)|^{2}$. Omitting the denominator we obtain

$$
\dot{z}=-p(z) \bar{q}(z) .
$$

The solution curves of (1.6) are the same as those of (1.1), with a different parametrization. The real and imaginary parts of $p \bar{q}$ are polynomials in $x$ and $y$.

A question about (1.5) of current interest (Hilbert's sixteenth problem) is that of obtaining an upper bound for the number of limit cycles of (1.5), in terms of the degrees of $X$ and $Y$. Ecalle, Martinet, Moussu, and Ramis [3, 4] and $\mathrm{Il}^{\prime}$ yashenko [6] have published research announcements that problem (1.5) can have only finitely many limit cycles. One of the results of this paper is that (1.3) can have no limit cycles. The first example of a quadratic system with four limit cycles was given by Shi Songling [9] in 1980. Other recent work on this problem can be found in [7] and references given there.

In the special case that there exists a polynomial $p(z)$ such that

$$
R(z)=-p(z) / p^{\prime}(z),
$$

each open set $U$ consists of the trajectories which converge to a fixed zero of $p$ in forward time and converge to $\infty$ in backward time. The boundary of these open sets consists of the trajectories which reach a pole of $R$ in finite 
time (either forward or backward). The Newton graph of $p$ consists of those trajectories reaching a pole of $R$ in backward time (see [10]).

The function $f$ of (1.2) is not in general a polynomial. It may have essential singularities, branch points, poles, and combinations of these. We describe the sets $U$ and the boundary trajectories in terms of properties of $f$.

The idea of finding the zeros of a (single-valued) function $F$ defined in $\mathbf{R}^{n}$ and taking values in $\mathbf{R}^{n}$, by following the trajectories of

$$
\dot{X}=-(D F(X))^{-1} F(X),
$$

appears in a number of recent papers $[11,5,12,13]$. In [11] $F$ is considered in an open and bounded set $M$ with a smooth boundary. The emphasis in that paper is on conditions on the boundary which guarantee that $F$ has a discrete zero set in $M$ and that most trajectories starting on the boundary converge to a point in the zero set. In $[5,12,13]$ the main concern is with algorithms and their efficiency in the implementation of the continuous Newton method. In [10] the Newton graph of a polynomial is characterized in graph-theoretical terms.

We adopt the following terminology: a zero of $R$ is an equilibrium solution of (1.1), and a zero of $f^{\prime}$ which is not also a zero of $f$ is a critical point of $f$. If $w=f(z)$, where $z$ is a critical point, then $w$ is a critical value of $f$, and the ray in the $w$-plane from the origin and through $f(z)$ is a critical ray. A trajectory of (1.3) which reaches a critical point in finite time is called a critical trajectory.

\section{IMPLICIT SOLUTIONS}

Assume the rational function $R$ has the form $R=-p / q$, where $p, q$ are polynomials with no common factors. Then $R$ is of the form (1.2) for any $f$ with logarithmic derivative

$$
f^{\prime}(z) / f(z)=q(z) / p(z) .
$$

The structure of all $f$ satisfying this differential equation is obtained by integrating the partial fractions expansion of $q / p$. Let

$$
p(z)=\prod_{j=1}^{J}\left(z-z_{j}\right)^{m_{j}}, \quad q(z)=\prod_{k=1}^{K}\left(z-s_{k}\right)^{n_{k}},
$$

where the $m_{j}$ and $n_{k}$ are positive integers and (without loss of generality) $p$ is monic.

2.3. Theorem. There exist unique polynomials $P_{j}, j=0, \ldots, J$ with $P_{j}(0)=$ 0 , and there exist unique complex constants $A_{j}, j=1, \ldots, J$, such that $f$ satisfies (2.1) if and only if

$$
f(z)=C e^{P_{0}(z)} \prod_{j=1}^{J}\left(z-z_{j}\right)^{A_{j}} e^{P_{j}\left(1 /\left(z-z_{j}\right)\right)}
$$

where $C$ is an arbitrary nonzero constant. 
Proof. Let $d(z)$ and $r(z)$ be polynomials, with the degree of $r$ less than the degree of $p$, such that $q / p=d+r / p$. Then $r / p$ has the partial fractions expansion

$$
\frac{r(z)}{p(z)}=\sum_{j=1}^{J}\left[\frac{A_{j}}{z-z_{j}}+\sum_{k=2}^{m_{j}} \frac{A_{j k}}{\left(z-z_{j}\right)^{k}}\right],
$$

where the sum from 2 to $m_{j}$ is zero if $m_{j}=1$. Let $P_{0}$ be the unique polynomial such that $P_{0}^{\prime}=d$ and $P_{0}(0)=0$, and let $P_{j}, 1 \leq j \leq J$, be the unique polynomials such that

$$
\left(P_{j}\left(\frac{1}{z}\right)\right)^{\prime}=\sum_{k=2}^{m_{j}} \frac{A_{j k}}{z^{k}}, \quad P_{j}(0)=0
$$

Then

$$
(\ln f(z))^{\prime}=P_{0}^{\prime}(z)+\sum_{j=1}^{J}\left[\left(\ln \left(z-z_{j}\right)^{A_{j}}\right)^{\prime}+\left(P_{j}\left(\frac{1}{z-z_{j}}\right)\right)^{\prime}\right] .
$$

We obtain (2.4) by integrating and exponentiating the above equation.

From (2.4) we see that $R$ is the Newton map of a function $f$ with an essential singularity at $\infty$ if and only if $R$ is bounded at $\infty$, and $R$ is the Newton map of an $f$ with an essential singularity in the finite plane if and only if $R$ has a multiple root. Consequently $R$ is the Newton map of a rational function if and only if $R$ is unbounded at $\infty$ and all of $R$ 's zeros are simple. $R$ is the Newton map of a polynomial if and only if it satisfies the further condition that $-1 / R^{\prime}\left(z_{j}\right)$ is a positive integer for each zero $z_{j}$ of $R$. Further, $f$ has at most finitely many branch points, at those $z_{j}$ for which $A_{j}$ is not an integer, and there are finitely many branch cuts. Since $f^{\prime}=f / R$, we see that each branch of $f$ has the same critical points, but there can be infinitely many distinct critical values and critical rays.

2.5. Example. Let $f(z)=z^{\sqrt{2}}(z-1)$. There is only one critical point, at $z_{c}=\sqrt{2} /(1+\sqrt{2})$, but there are infinitely many critical values, dense on a circle centered at the origin of the $w$-plane.

Also from (2.4), we can compute the vector field $R(z)$ in a neighborhood of an equilibrium solution. Assuming, without loss of generality, that $z=0$ is an equilibrium solution, $f$ can then be written as

$$
f(z)=z^{a} e^{P(1 / z)} \psi(z)
$$

where $a$ is an arbitrary complex constant, $P(\zeta)$ is either identically zero or has the form

$$
P(\zeta)=A_{1} \zeta+\cdots+A_{m} \zeta^{m}, \quad A_{m} \neq 0
$$


and $\psi$ is analytic and nonzero in a neighborhood of zero. Then

$$
-\frac{f(z)}{f^{\prime}(z)}=-1 /\left(\frac{a}{z}-\frac{1}{z^{2}} P^{\prime}\left(\frac{1}{z}\right)+\frac{\psi^{\prime}(z)}{\psi(z)}\right),
$$

so that the differential equation (1.3) takes the form

$$
\dot{z}=-1 /\left(\frac{-m A_{m}}{z^{m+1}}-\cdots-\frac{A_{1}}{z^{2}}+\frac{a}{z}+\sum_{0}^{\infty} c_{k} z^{k}\right) .
$$

Since at least one of the coefficients $a, A_{m}$ is not zero, (1.3) takes the form

$$
\dot{z}=b z^{m+1}(1+\phi(z)),
$$

where $b$ is a nonzero constant, $\phi$ is analytic at zero, $\phi(0)=0$, and $m$ is an integer which is at least 1 if $P$ is not identically zero and is 0 otherwise. Thus a degenerate equilibrium solution arises from an essential singularity in $f$. For $m \geq 1$ the role of $b$ is to determine the orientation of the picture, since replacing $z$ with $\alpha z$ changes $b$ into $\alpha^{m} b$. For $m=0$ this has no effect, and in this case the value of $\operatorname{Re}(b)$ determines the character of the equilibrium solution of the linearized problem.

Information about the global nature of solutions to (1.3) can be obtained from the implicit solution

$$
f(z(t))=f(z(0)) e^{-t}
$$

which is interpreted as applying to each single-valued branch of $f$.

2.11. Definition. A solution of (1.3) is a continuous curve $z(t),-\infty<t<\infty$, such that for every finite time interval $[a, b]$, there is a partition $a=\tau_{0}<\tau_{1}<$ $\cdots<\tau_{n}=b$, and there exist single-valued branches $f_{1}, \ldots, f_{n}$ of $f$, such that

$$
\begin{aligned}
& f_{1}(z(t))=f_{1}\left(z\left(\tau_{0}\right)\right) e^{-\left(t-\tau_{0}\right)}, \quad \tau_{0} \leq t \leq \tau_{1}, \\
& f_{j}(z(t))=f_{j-1}\left(z\left(\tau_{j-1}\right)\right) e^{-\left(t-\tau_{j-1}\right)}, \quad \tau_{j-1} \leq t \leq \tau_{j},
\end{aligned}
$$

for $2 \leq j \leq n$.

A solution which avoids the critical points of $f$ for all time is an ordinary solution, and a solution which passes through a critical point is a generalized solution. A solution $z(t)$ is periodic if there exists $\omega>0$ such that $z(t+\omega)=$ $z(t)$ for all $t$. If $z(t)$ is a periodic generalized solution then it may consist of more than one closed loop. (See Example 2.15 and Figure 1.)

From this definition we see that a solution is a continuous curve which can be mapped continuously onto a ray $w=w_{0} e^{-t}$ by a succession of branches of $f$. By the implicit function theorem, a solution has a continuous derivative $\dot{z}(t)$ as long as $z(t)$ is not a critical point of $f$, and then $\dot{z}(t)=R(z(t))$ and there is only one solution through any noncritical point. If $z_{c}$ is a critical point of $f$, and $k \geq 2$ is the first integer such that $f^{(k)}\left(z_{c}\right) \neq 0$, then there are $k^{2}$ solutions through $z_{c}$, with corners at $z_{c}$ which make angles which are integer multiples of $\pi / k$. Note that $\dot{z}(t)$ is continuous across the branch cuts of $f$. 
2.12. Example. Let $f(z)=z^{2}-1$ and $R(z)=-\left(z^{2}-1\right) / 2 z$. Let $\sqrt{\zeta}$ be defined to have its argument in $(\pi / 2, \pi / 2]$. Then

$$
z(t)= \begin{cases}\sqrt{1-2 e^{-t}}, & -\infty<t \leq \log 2, \\ -\sqrt{1-2 e^{-t}}, & \log 2 \leq t<\infty,\end{cases}
$$

is a continuous curve making a right-angle turn as it passes through 0 at time $t=\log 2$, and $f(z(t))=f(z(0)) e^{-t}$. Thus this trajectory consists of the positive imaginary axis, traversed towards the origin as $t$ increases to $\log 2$, and then of the segment of the negative real axis between -1 and 0 , traversed towards -1 .

2.13. Example. Let $f(z)=z^{-i}$ and $R(z)=-i z$. Then $f$ has no critical points, but it has branch points at 0 and $\infty$, and a branch cut which we take to be the negative real axis. Then the $k$ th branch of $f$ is related to the principal branch by $f_{k}(z)=e^{2 k \pi} f_{0}(z)$. If $\operatorname{Arg} z(0)=\theta_{0}$, let $\tau_{k}=\theta_{0}+\pi(2 k+1)$. Then for the solution $z(t)=z(0) e^{-i t}$, we have

$$
f_{k}(z(t))=f_{k-1}\left(z\left(\tau_{k-1}\right)\right) e^{-\left(t-\tau_{k-1}\right)} .
$$

A consequence of the definition of a solution is that $|f(z(t))| \rightarrow 0$ as $t \rightarrow \infty$ and $|f(z(t))| \rightarrow \infty$ as $t \rightarrow-\infty$, but, as we see in the previous example, this does not imply that $z(t)$ has a limit.

Note that if $z_{c}$ is a critical point of $f$, and if $f_{0}$ is any single-valued branch of $f$, then $f_{0}\left(z_{c}\right)$ is an algebraic branch point of $f_{0}^{-1}$.

2.14. Theorem. For (1.3) to have a periodic solution, it is necessary that each integer power of $f$ be multivalued.

Proof. By (2.10), the map $\mathbf{R} \rightarrow \mathbf{C}$ defined by $t \rightarrow f^{n}(z(t))$ is one-to-one. If $z(t)$ is periodic there exists $\omega>0$ such that $z(0)=z(\omega)$. Thus $f^{n}$ has more than one branch defined at $z(0)$.

We now consider the general case that $f$ is defined on an infinitely sheeted Riemann surface. We show that any trajectory bounded away from the equilibrium solutions of (1.3) must be periodic.

2.15. Example. Let $f(z)=\left(z^{2}-1\right)^{i / 2}$ and $R(z)=i(z-1 / z)$. The only critical point is at $z=0$ and the positive real axis is the critical ray. Each interval $\left[e^{-\pi / 2} e^{-k \pi}, e^{-\pi / 2} e^{-(k-1) \pi}\right]$ is mapped by $f^{-1}$ onto a figure eight in the $z$-plane which crosses at $z=0$ (see Figure 1).

2.16. Theorem. Let $\phi(t)$ be a solution of (1.3) which is contained in a compact subset $K$ of $\mathbf{C}$, where $K$ contains no equilibrium solutions of (1.3). Then there exists $\omega>0$ such that $\phi(t+\omega)=\phi(t)$ for all $t$.

Note that the Poincaré-Bendixson theorem cannot be applied, since solutions are not unique. Also, a consequence of Theorem 2.16 is that (1.3) has no limit cycles. 
The proof of Theorem 2.16 has two parts. The first is to show that if $\tilde{z}$ is a point in $K$ which is in the forward limit set of $\phi$, then there is a number $\omega>0$ and there is a solution $\gamma(t)$ through $\tilde{z}$ such that $\gamma(t+\omega)=\gamma(t)$. The second part is to show that $\gamma$ and $\phi$ coincide. Without loss of generality, we assume $\phi$ is free of critical points in forward time, since otherwise $\phi$ would be a graph connecting a subset of the finitely many critical points of $f$, thus consisting of closed loops.

2.17. Lemma. Assume $\tilde{z}$ is in the forward limit set of $\phi$, but that $\tilde{z}$ is not in $\phi$. Then there exists a simple open $C^{1}$ curve $\Gamma$, with $\tilde{z}$ at one end, such that $\phi(t)$ crosses $\Gamma$ at right angles for a sequence of times $\rightarrow \infty$.

Proof. Let $f_{1}$ be a single-valued branch of $f$, analytic in a neighborhood of $\tilde{z}$, let $\tilde{w}=f_{1}(\tilde{z})$, and let $\tilde{\rho}$ denote the ray from the origin passing through $\tilde{w}$. Since $\tilde{z}$ is in the forward limit set of $\phi$, there exists a sequence $t_{n} \rightarrow \infty$ such that $\phi\left(t_{n}\right) \rightarrow \tilde{z}$. Then $f_{1}\left(\phi\left(t_{n}\right)\right) \rightarrow \tilde{w}$. We assume, without loss of generality, that all of the points $f_{1}\left(\phi\left(t_{n}\right)\right)$ lie on one side of $\tilde{\rho}$. If $\tilde{\lambda}$ is the angle of $\tilde{\rho}$, let $R$ denote the polar rectangle consisting of $w$ such that

$$
|\tilde{w}| e^{-\delta}<|w|<|\tilde{w}| e^{\delta}, \quad \tilde{\lambda}<\operatorname{Arg} w<\tilde{\lambda}+\varepsilon,
$$

for some $\varepsilon, \delta>0$. Thus we assume $f_{1}\left(\phi\left(t_{n}\right)\right)$ is in $R$. Let $\Lambda$ denote that portion of the circular arc $|w|=|\tilde{w}|$ in $R$. Then $\Lambda$ is orthogonal to each ray, and if $g_{1}$ is the branch of $f_{1}^{-1}$ such that $g_{1}\left(f_{1}\left(\phi\left(t_{n}\right)\right)\right)=\phi\left(t_{n}\right)$ and $g_{1}(\tilde{w})=\tilde{z}$, then let $\Gamma=g_{1}(\Lambda)$. Note that $\Gamma$ is orthogonal to any trajectory of (1.3), except at $\tilde{z}$ if $\tilde{z}$ is a critical point of $f$ and then $g_{1}$ is not conformal. (Refering to Example 2.15 and Figure 1, we see that if $\tilde{z}$ is the critical point at 0 , then there are four solutions through this point depending on the choice of the branch of $f_{1}^{1}$, and all make right angle turns.) Since $g_{1}$ maps those segments of the rays containing $f_{1}\left(\phi\left(t_{n}\right)\right)$ in $R$ into the unique trajectories through $\phi\left(t_{n}\right)$, and since the ray segments intersect $\Lambda$, we see that $\phi$ intersects $\Gamma$ infinitely often. This ends the proof of the lemma.

Thus we assume, without loss of generality, that the points $\phi\left(t_{n}\right)$ are all distinct and converge to $\tilde{z}$ on $\Gamma$, and that $t_{n}$ is the first return to $\Gamma$ after time $t_{n-1}$. Let $J_{n}$ denote the Jordan curve consisting of $\phi(t)$ for $t_{n} \leq t \leq t_{n+1}$ and also consisting of that portion of $\Gamma$ between $\phi\left(t_{n}\right)$ and $\phi\left(t_{n+1}\right)$. Let $B_{n}$ denote that part of the plane interior to $J_{n}$. There are two cases, depending on whether the continuation of $\phi(t)$ for $t>t_{n+1}$ is exterior or interior to $B_{n}$. In the first case $B_{n}$ is a monotonically increasing sequence of sets and in the second it is a monotonically decreasing sequence of sets. Let $B_{\infty}$ denote the intersection of the sets $B_{n}$. Since $f$ has at most finitely many critical points and branch points, we assume, without loss of generality (by selecting $t_{1}$ sufficiently large), that any such points which are in $B_{n}$ for some $n$ are already in $B_{\infty}$. Then $B_{n}-B_{\infty}$ is free of critical points and branch points for $n \geq 2$. Thus in each time interval $\left[t_{n}, t_{n+1}\right], \phi(t)$ crosses the same finite sequence of branch cuts of $f$. Let $f_{1}, \ldots, f_{N}$ be a sequence of single-valued branches of $f$, mapping 
$\phi(t), t_{n} \leq t \leq t_{n+1}$, into a ray through $f_{1}\left(\phi\left(t_{n}\right)\right)$. This sequence of branches of $f$ is independent of $n$.

2.18. Lemma. Let $\omega=t_{2}-t_{1}$. Any trajectory on $\Gamma$ at time $t=0$ is on $\Gamma$ at time $t=\omega$.

Proof. Let $g_{N}$ be that branch of $f_{N}^{-1}$ such that $g_{N}\left(f_{N}\left(\phi\left(t_{2}\right)\right)\right)=\phi\left(t_{2}\right)$. Then $g_{N}\left(\Lambda e^{-\omega}\right)$ is the orthogonal trajectory through $\phi\left(t_{2}\right)$, i.e., $\Gamma$. This ends the proof of the lemma.

We can assume, without loss of generality, that the finitely many branch cuts crossed by $\phi$ are orthogonal trajectories, since a branch cut can be continuously deformed as long as it does not cross another branch cut or branch point, and there are no branch points inside of $B_{n}$ for $n \geq 2$. Assuming trajectories start on $\Gamma$ at time $t=0$, we have a sequence of times $0<\beta_{1}<\cdots<\beta_{N}<\omega$ such that all trajectories on $\Gamma$ cross the branch cuts at these times.

We now construct the solution $\gamma$. For that portion of $\tilde{\rho}$ which is part of the boundary of $R$, let $\gamma(t)=g_{1}(\tilde{\rho})$, where $\tilde{\rho}$ is exponentially parametrized. Then $\gamma(t)$ is a segment of a (possibly nonunique) solution through $\tilde{z}$ which has a unique extension in forward time (also denoted $\gamma(t)$ ) until it reaches a critical point of $f$. If a critical point is reached before $t=\beta_{1}$, then a possibly different branch of $f_{1}^{-1}$ is selected, so that the rays through the points $f_{1}\left(\phi\left(t_{n}\right)\right)$ are mapped continuously in the $z$-plane. As $\gamma$ crosses a branch cut of $f$, the appropriate branch of $f_{n}^{-1}$ is selected, $1 \leq n \leq N$. Thus $\gamma(t)$ is uniquely defined for $0 \leq t \leq \omega$, and $\gamma(\omega)=\gamma(0)=\tilde{z}$, since $\gamma(\omega)$ is on the closure of $g_{N}\left(\Lambda e^{-\omega}\right)$. Since $g_{N}$ maps the portion of $\tilde{\rho}$ near $\tilde{w} e^{-\omega}$ onto $\gamma$, we see that $\gamma(t+\omega)=\gamma(t)$ for all $t$.

Finally, we show that $\gamma$ and $\phi$ coincide, by showing that the assumption that the points $\phi\left(t_{n}\right)$ are all distinct leads to a contradiction. Since the sets $B_{n}-B_{\infty}$ are free of critical points of $f$, the flow $T_{t}$ defined by the differential equation is analytic in forward time, if it starts on $W=g_{1}(R)$, and it has a continuous extension to that portion of $\tilde{\rho}$ which is part of the boundary of $R$. Let $h(z)$ be a polynomial, and let

$$
h_{\omega}(z)=h\left(T_{-\omega}(z)\right), \quad z \text { in } T_{\omega}(W) .
$$

Since $T_{-\omega} \phi(k \omega)=\phi((k-1) \omega)$, we have $h_{\omega}(\phi(k \omega))=h(\phi((k-1) \omega))$. Since $W$ and $T_{\omega}(W)$ have nonempty intersection and have a common boundary consisting of a portion of $\gamma(t)$, we see that $h$ and $h_{\omega}$ are analytic continuations of each other. Thus all polynomials are constant on $\phi(k \omega)$. This contradicts the assumption that the points $\phi(k \omega)$ are all distinct, so for $\tilde{z}$ to be in the forward limit set of $\phi$, it is necessary that $\tilde{z}$ be in $\phi$.

\section{Solutions AT AN ESSENTIAL SINGULARITY}

We consider (2.9) with $m$ at least one. An essential singularity serves as both a source and a sink, in the sense that there is a "basin of attraction" in each time direction. Clearly the essential singularity cannot be interior to either 


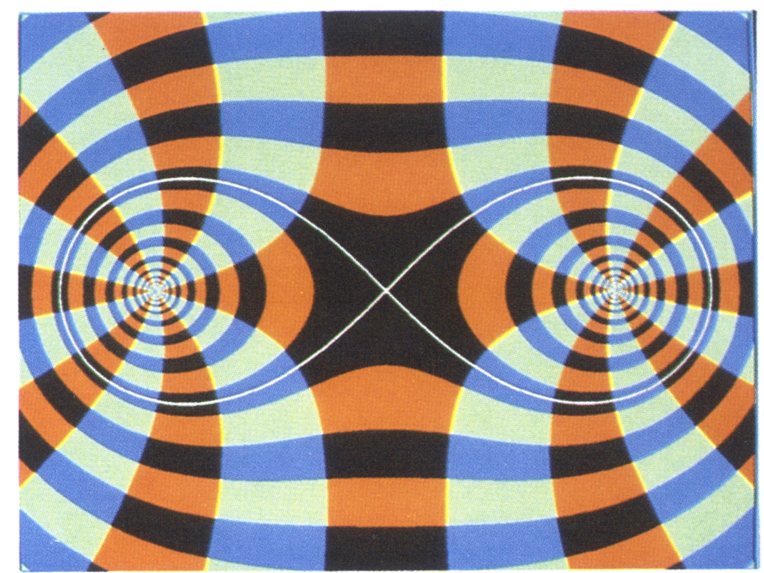

FIGURE 1. $f(z)=\left(z^{2}-1\right)^{i / 2}$

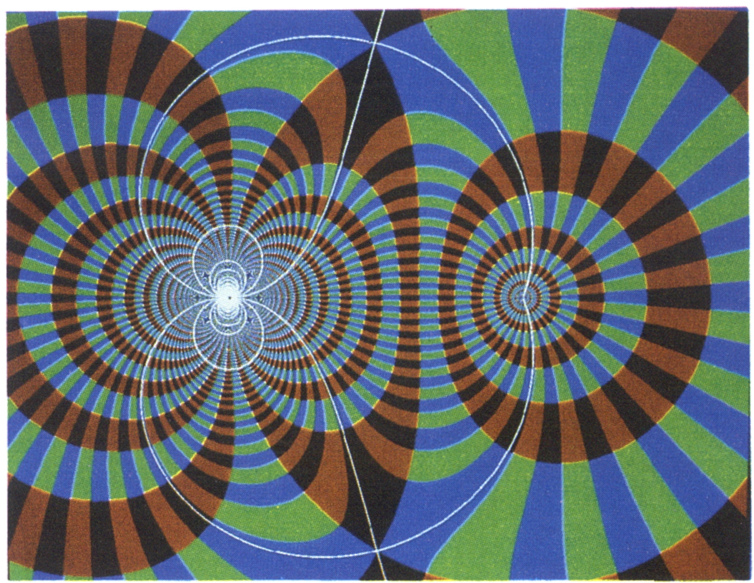

Figure 2. $f(z)=e^{1 / z}(z-1)$

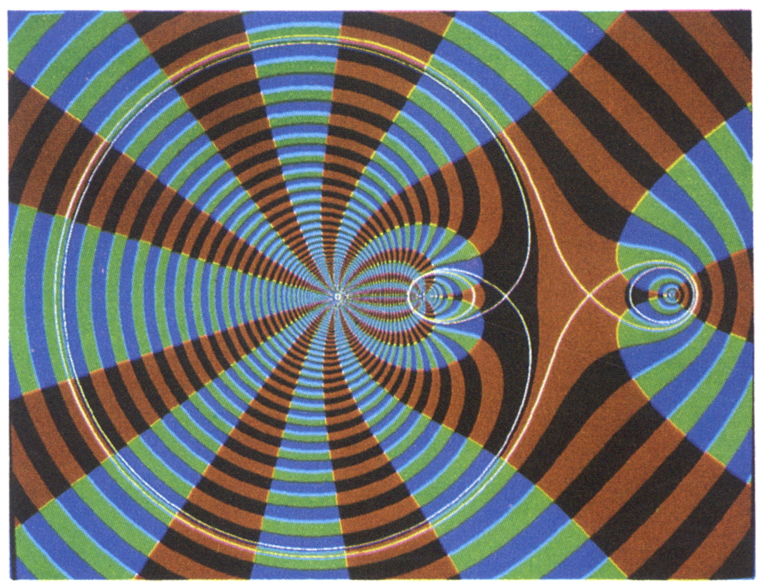

Figure 3. $f(z)=(z+1)^{3 i / 2} z^{-2 i / 3}(z-3)^{i / 6}$ 
of its basins. We show that a characteristic feature of this case is the existence of solutions, existing for all time, which converge to the essential singularity in both forward and backward time, tangent to fixed directions.

3.1. Example. $\dot{z}=-z^{2}$, the continuous Newton method of $f(z)=e^{1 / z}$. The solution curves are circles, tangent to the real axis at zero. The real axis is itself a solution curve through $\infty$.

3.2. Example. $\dot{z}=-z^{3} / 2$, the continuous Newton method of $f(z)=e^{1 / z^{2}}$. The ordinary solutions are loops in each quadrant, emanating from the origin tangent to the real axis and returning to the origin tangent to the imaginary axis. The positive and negative real axes are critical trajectories, reaching $\infty$, which is a singularity of the vector field, in finite time, and then returning to the origin along the positive and negative parts of the imaginary axis.

3.3. Example. $\dot{z}=-z^{2}(z-1) /\left(z^{2}-z+1\right)$, the continuous Newton method of $f(z)=e^{(1 / z)}(z-1)$. The factor $z-1$ introduces a sink at 1 and a source at $\infty$. In addition to trajectories connecting 0 to 0 there are trajectories connecting $\infty$ to $0, \infty$ to 1 , and 0 to 1 . The vector field has singularities at the zeros of $z^{2}-z+1$ (see Figure 2).

For the general study of (2.9) we consider a disk $D=\{z:|z|<\mu\}$, where $|\phi(z)| \leq K|z|$ and $\phi$ has no other zeros in $D$, and we consider a sector $S$ in $D$ of angular opening $2 \pi / m: \alpha \leq \arg z \leq \alpha+2 \pi / m$. The change of variable

$$
w=1 / m b z^{m}
$$

maps $S$ onto a neighborhood $D_{w}$ of $\infty$ in the $w$-plane: $|w|>\nu$, for some $\nu>0$, and the boundary rays of $S$ correspond to the ray $\arg w=m \alpha$. Let

$$
q(w)=\phi\left((1 / m b w)^{1 / m}\right),
$$

where the $m$ th root is selected to be in $S$. Then

$$
|q(w)| \leq K|w|^{1 / m}
$$

in $D_{w}$, and

$$
\dot{w}=-1+q(w) .
$$

We consider (3.7) with initial condition

$$
w(0)=w_{0}, \quad w_{0} \text { in } D_{w} .
$$

Note that $\dot{w}=-1$ is the continuous Newton method for $f(w)=e^{w}$. A solution of (3.7), (3.8) will fail to exist for all forward time if one of the following three events occurs:

(1) it goes to $\infty$ in finite time;

(2) it reaches $|w|=\nu$ in finite time;

(3) it reaches the branch cut $\arg w=m \alpha$ in finite time.

Assume $\alpha=0$, so that the branch cut is along the positive real axis. 
3.9. Lemma. Let $R>0$ be selected so that $R^{m}>\nu$. If

$$
\left|w_{0}\right|>R^{m}
$$

then as long as $|w(t)|>R^{m}$, we have

$$
\left|w(t)-w_{0}+t\right| \leq \frac{K}{R} t
$$

Proof. As long as it exists, $w(t)$ satisfies the integral equation

$$
w(t)=w_{0}-t+\int_{0}^{t} q(w(s)) d s .
$$

The result follows from (3.6) and (3.10).

Assuming further that $\frac{K}{R}<1$, let $\beta=\beta(R)$ be that number in $(0, \pi / 2)$ such that $\sin \beta=\frac{K}{R}$. Let $T\left(w_{0}, R\right)$ denote the sector in the $w$-plane with vertex at $w_{0}$, angular opening $2 \beta$, and bisecting line $w_{0}-t$ for $t \geq 0$ :

$$
T\left(w_{0}, R\right)=\left\{w:\left|w-w_{0}+t\right| \leq \frac{K}{R} t\right\} .
$$

Then Lemma 3.9 can be refined to:

3.14. Lemma. Let $R>0$ be selected so that $R^{m}>\nu$ and $R>K$. If $\left|w_{0}\right|>$ $R_{m}$, then as long as $|w(t)|>R^{m}, w(t)$ is in $T\left(w_{0}, R\right)$.

Let $U_{+}(R)$ denote the open set consisting of $w$ 's such that (1) $|w|>R^{m}$ and (2) if $\operatorname{Re} w \geq-R^{m} \frac{K}{R}$, then

$$
|\operatorname{Im} w|>R^{m} \sec \beta+(\operatorname{Re} w) \tan \beta .
$$

Let $C_{+}(R)$ denote the closed set which is the complement of $U_{+}(R)$ in the $w$-plane.

3.15. Theorem. If $w_{0}$ is in $U_{+}(R)$, then the solution $w(t)$ of (3.7), (3.8) exists and is in $U_{+}(R)$ for all forward time, it is unbounded as $t \rightarrow \infty$, and $|\operatorname{Arg} w(t)| \rightarrow \pi$.

Proof. If $w_{0}$ is in $U_{+}(R)$, then $T\left(w_{0}, R\right)$ is in $U_{+}(R)$ so $|w(t)|>R^{m}$ for as long as it exists. By (3.11), w(t) cannot become unbounded in finite time, and since the circle $|w|=\nu$ and the branch cut are both in $C_{+}(R), w(t)$ must exist for all forward time.

Also from (3.11), $|w(t)| \geq\left|w_{0}-t\right|-\frac{K}{R} t$. Since $\frac{K}{R}<1$ we can select $t$ so large that $\left|1-w_{0} / t\right|-\frac{K}{R}>\kappa$ for some $\kappa>0$. Then $|w(t)|>\kappa t$ which becomes arbitrarily large as $t \rightarrow \infty$.

To investigate $\operatorname{Arg} w(t)$, we note that $|\operatorname{Arg} w(t)-\pi|<\beta+\varepsilon$ in the sector $T\left(w_{0}, R\right)$, for each $\varepsilon>0$ and $t$ sufficiently large. Note that if $\frac{\pi}{2}+\beta \leq$ $\operatorname{Arg} w_{0} \leq w_{0} \leq 3 \frac{\pi}{2}-\beta$ then $|w| \geq\left|w_{0}\right|$ for all $w$ in $T\left(w_{0}, R\right)$. Select $t_{0}>0$ such that $\operatorname{Re} w\left(t_{0}\right) \leq-R^{m} \frac{K}{R}$. Then $T\left(w\left(t_{0}\right),\left|w\left(t_{0}\right)\right|\right)$ is contained in $U_{+}\left(\left|w\left(t_{0}\right)\right|\right)$, so

$$
|\operatorname{Arg} w(t)-\pi|<\beta\left(\left|w\left(t_{0}\right)\right|\right)
$$


for $t \geq t_{0}$. Since $\beta(R) \rightarrow 0$ as $R \rightarrow 0$, we see that

$$
\lim _{t \rightarrow \infty}|\operatorname{Arg} w(t)|=\pi \text {. }
$$

3.17. Definition. A curve $w(t)$ is asymptotically parallel to the negative real axis if (3.16) holds and $\lim _{t \rightarrow \infty}|w(t)|=\infty$.

Let $U_{+}=\bigcup U_{+}(R)$, where the union is taken over all $R$ such that $R^{m}>\nu$ and $\frac{K}{R}<1$. Let $C_{+}$be the complement of $U_{+}$. Then $U_{+}$is an open set and any initial point $w_{0}$ in $U_{+}$generates a solution $w(t)$ which is asymptotically parallel to the negative real axis. We show that all solutions which are unbounded in forward time must enter $U_{+}$, and thus are asymptotically parallel to the negative real axis.

To consider solutions of (3.7), (3.8) which are near the positive real axis, let $S$ now denote the sector $-\pi / m<\operatorname{Arg} z \leq \pi / m$. Then the branch cut is along the negative real axis.

3.18. Lemma. Let $L(P)$ denote the vertical line $\operatorname{Re} w=P$. There exists $P_{0}>$ 0 such that if $P>P_{0}$ and if $w(t)$ is the solution of (3.7), (3.8) with $\operatorname{Re} w_{0}=P$, then $\operatorname{Re} w(t) \leq P$ for as long as $w(t)$ exists.

Proof. From (3.12),

$$
\operatorname{Re} w(t)=\operatorname{Re} w_{0}-t+\operatorname{Re} \int_{0}^{t} q(w(s)) d s .
$$

Let $K / P_{0}<1$. Then $K / P<1$ and as long as $\operatorname{Re} w(t)>P_{0}$,

$$
\operatorname{Re} w(t)<P-t+K / P t<P .
$$

Since (3.7) is autonomous, this holds for any point on the trajectory which is also on $L(P)$.

3.20. Corollary. Let $\varepsilon>0$ be given, $0<\varepsilon<\pi / 2$. Any solution of (3.7) which exists for all forward time and is unbounded must eventually satisfy $|\operatorname{Arg} w(t)|>$ $\varepsilon$.

Proof. The triangle $|\operatorname{Arg} w| \leq \varepsilon, \operatorname{Re} w \leq P$ is bounded, so if $w_{0}$ is in the triangle then $w(t)$ must eventually leave it. By the previous lemma, $w(t)$ cannot cross the line $\operatorname{Re} w=P$ if $P$ is sufficiently large.

3.21. Theorem. Any solution of (3.7), (3.8) which exists for all forward time and is unbounded must be in $U_{+}$for sufficiently large $t$.

Proof. Any such solution $w(t)$ must eventually leave a sector containing the positive real axis, so without loss of generality we can assume the solution will not cross that axis. Since that part of $C_{+}$to the left of any line $L(P)$ is bounded, we see that $w(t)$ must eventually be in $U_{+}$.

3.22. Lemma. Let $\varepsilon>0$ be given. There exists $R(\varepsilon)>0$ such that if $w_{0}$ is in $C_{+}$and $\left|w_{0}\right|>R(\varepsilon)$, then $\left|w_{0}\right|<\varepsilon$. 
Proof. Since $\sin \beta=\frac{K}{R}$, we can select $R$ so large that $\beta<\varepsilon$. The line tangent to the circle of radius $R$ at the point of angle $\pi / 2+\beta$ intersects the ray of angle $\varepsilon$ at a point a distance $R(\varepsilon)$ from the origin.

All of the above considerations apply to solutions which exist and are unbounded in backward time.

3.23. Theorem. There exists an open set $U_{-}$such that all solutions of (3.7), (3.8) which exist for all backward time and are unbounded must eventually be in $U_{-}$. Such solutions are asymptotically parallel to the positive real axis in the w-plane.

We now interpret these results in the $z$-plane. Assume that $b=1$ in (2.9) and (3.4). Let $V_{ \pm}$be the image of $U_{ \pm}$under the (multivalued) inverse of (3.4).

3.24. Theorem. A solution of (2.9) converges to 0 if and only if it is eventually in $V_{+}$, and then it approaches 0 tangent to a ray $\operatorname{Arg} z=(2 k+1) \pi / m$, $k=0, \ldots, m-1$. A solution which converges to zero in backward time must eventually be in $V_{-}$, and it approaches 0 tangent to a ray $\operatorname{Arg} z=2 j \pi / m$.

\section{Almost linear EQUilibria}

If $P$ is identically zero in (2.6), then (2.9) takes the form

$$
\dot{z}=-\frac{1}{a} z(1+\phi(z)) .
$$

If $\operatorname{Re} a \neq 0$ then the standard theory of perturbations of linear systems holds.

4.2. Theorem. If $\operatorname{Re} a>0$ then the equilibrium solution at 0 is asymptotically stable in forward time, and there exists an open set $U$ containing 0 such that the trajectory $z(t)$ converges to 0 in forward time if and only if $z(t)$ eventually enters $U$. If $\operatorname{Re} a<0$ then the same situation holds in backward time.

For the case $\operatorname{Re} a=0$ we disregard inessential constants and consider

$$
\begin{gathered}
f(z)=z^{i}(1+z \phi(z)), \\
\dot{z}=i z(1+z \phi(z)),
\end{gathered}
$$

where $\phi(z)$ is a generic function, analytic at 0 . The expression $z^{i}$ on a trajectory $z(t)$ is evaluated by selecting $z(0)=r(0) e^{i \theta(0)}$, where $-\pi<\theta(0) \leq \pi$, and then $\theta(t)$ is selected to vary continuously on $z(t)$. Thus

$$
z^{i}(t)=e^{-\theta(t)} e^{i \log r(t)} .
$$

We show that all orbits starting sufficiently close to 0 are periodic. Note that the unperturbed differential equation $\dot{z}=i z$ is Hamiltonian, but no perturbed equation can be Hamiltonian. 
4.5. Lemma. No solution of (4.4) can converge to 0 in forward or backward time.

Proof. Let $z=r e^{i \theta}$ be a trajectory starting at $z_{0}=r_{0} e^{i \theta_{0}}$, and assume this trajectory is an ordinary solution converging to 0 in forward time. Let $s=$ $\log |z|$. The implicit solution (2.9) takes the form

$$
e^{-\theta} e^{i s}(1+z \phi(z))=e^{-\theta_{0}-t} e^{i s_{0}}\left(1+z_{0} \phi\left(z_{0}\right)\right) .
$$

Then

$$
e^{i s}(1+z \phi(z))=m(t) e^{i \alpha}
$$

for some positive function $m(t)$ and fixed angle $\alpha$. If $z \phi(z) \rightarrow 0$ as $t \rightarrow \infty$, then $s(t) \rightarrow \alpha+2 k \pi$ for a fixed $k$, since $s(t)$ is continuous. Since $s=\log |z|$, a limit for $s$ is inconsistent with $|z| \rightarrow 0$. The same argument holds in the negative direction.

To show that solutions starting sufficiently close to the origin wind around it, we use (4.4) in polar coordinates:

$$
\dot{r}+i r \dot{\theta}=i r+i r^{2} e^{i \theta} \phi(z) .
$$

Let $i e^{i \theta} \phi(z)=a(z)+i b(z)$. Then

$$
\begin{gathered}
\dot{r}=r^{2} a(z), \\
\dot{\theta}=1+r b(z) .
\end{gathered}
$$

For a sufficiently small disk $|z|<\rho$, there exists a constant $K>0$ such that $|a(z)|,|b(z)| \leq K$. Without loss of generality, assume $\rho K<1$. From (4.6), $\left|\dot{r} / r^{2}\right| \leq K,-K \leq \frac{d}{d t}(-1 / r) \leq K$, and then $r(t) \leq\left(1 / r_{0}-K t\right)^{-1}$. In particular, $r(t)$ exists and satisfies $r(t)<\rho$ as long as $t<\left(1 / r_{0}-1 / \rho\right) / K$. Thus preassigning $T>0, r(t)$ exists up to $T$ for $r_{0}$ sufficiently small.

Now consider (4.7). We have $|\dot{\theta}-1|<\rho K$ as long as $r<\rho$. Assume $\theta_{0}=0$, so that the trajectory starts on the positive real axis. Then $\theta(t)>(1-\rho K) t$, and $\theta$ assumes the value $2 \pi$ if $t>2 \pi /(1-\rho K)$, which can be assured if $r_{0}$ is so small that

$$
\frac{2 \pi}{1-\rho K}<\frac{1}{K}\left(\frac{1}{r_{0}}-\frac{1}{\rho}\right) .
$$

Thus any trajectory starting on the positive real axis, sufficiently close to the origin, must return to the positive real axis.

4.8. Theorem. Any solution of (4.4) starting sufficiently close to 0 must be periodic.

Proof. Assume that a trajectory starting on the positive real axis makes its first return to the left of its starting point. Then the trajectory is spiraling inward in forward time, and since there are no critical points of $f$ close to the origin, the vector field is smooth in a neighborhood of the origin. By the PoincareBendixson theorem [2, p. 391], this trajectory must either converge to the equilibrium solution at 0 or cycle to a periodic solution. Since neither of these possibilities can occur, the trajectory must be periodic. 
4.9. Corollary. The set $U$ of points in the extended plane lying on ordinary periodic trajectories around 0 is an open set.

Proof. By the continuous dependence of solution on initial conditions, any trajectory starting sufficiently close to an ordinary periodic one must circle the origin, and if it is not periodic, then it must spiral towards a periodic solution in either forward or backward time, contradicting Theorem 2.16. (See Example 5.5 and Figure 3.)

\section{Global behavior}

We have seen that associated to each equilibrium solution $\tilde{z}$ of $(1.3)$ is an open set $\widetilde{V}$. If $\tilde{z}$ is asymptotically stable in forward time or is an essential singularity, then a trajectory converges to $\tilde{z}$ in forward time if and only if the trajectory enters $\widetilde{V}$. If $\tilde{z}$ is asymptotically stable in backward time, then a similar statement holds. If $\tilde{z}$ is a center, then each solution in $\widetilde{V}$ is an ordinary solution, is periodic, and encloses $\tilde{z}$. If $\tilde{z}$ is asymptotically stable in forward time or is an essential singularity, let $\widetilde{U}$ consist of those points on ordinary solutions which enter $\widetilde{V}$. Similarly for an equilibrium solution which is asymptotically stable in backward time. If $\tilde{z}$ is a center, let $\widetilde{U}$ denote the set of all points on ordinary solutions which are periodic and enclose $\tilde{z}$.

5.1. Theorem. For any equilibrium solution $\tilde{z}, \tilde{U}$ is an open set which is invariant under the differential equation.

Proof. Since all solutions are ordinary solutions, this comes from continuous dependence on initial data.

5.2. Theorem. A solution $z(t)$ of (1.3) which exists for forward time must be in $\widetilde{U}$ for some equilibrium solution $\tilde{z}$.

Proof. Suppose $z(t)$ is an ordinary solution not in any $\widetilde{U}$. Then $z(t)$ is not periodic and it is in a closed set $K$, free of equilibrium solutions. Without loss of generality, we assume $K$ is compact. By Theorem 2.16, $z(t)$ must be periodic, a contradiction.

5.3. Corollary. If $\tilde{z}$ is an equilibrium solution, then any point on the boundary of $\widetilde{U}$ is either a critical point or is on a trajectory which converges to a critical point in finite time.

5.4. Corollary. If $\tilde{z}$ is an equilibrium solution of (1.3), then each connected component of $\widetilde{U}$ is the largest connected open set containing a point of $\widetilde{U}$ which is free of critical trajectories.

Clearly $U_{j}$ is connected if $z_{j}$ is asymptotically stable in either direction. If $z_{j}$ is an essential singularity of $f$, then $U_{j}$ may be connected $\left(f(z)=e^{z}\right)$ or not $\left(f(z)=e^{z^{2}}\right)$. If $z_{j}$ is a center, then $U_{j}$ has a unique simply connected component $U_{j^{0}}$ which contains $z_{j}$, and all periodic orbits in $U_{j^{0}}$ enclose only $z_{j}$. 


\subsection{Example. Let}

$$
f(z)=(z+1)^{3 i / 2} z^{-2 i / 3}(z-3)^{i / 6} .
$$

Then

$$
R(z)=\frac{i(z+1) z(z-3)}{(z-1)(z-2)} .
$$

There are centers at $-1,0,3$, and $\infty$ and critical points at 1 and 2. Trajectories passing between 1 and 2 encircle the centers at -1 and 0 , as well as the centers at 3 and $\infty$ (see Figure 3 ).

We see from this example that an open set of periodic solutions is not necessarily associated with a unique center.

\section{NUMERICAL ANALYSIS AND PHASE PLANE PORTRAITS}

Phase plane portraits for autonomous systems often consist of curves obtained by eliminating the time parameter, thereby eliminating important information about the evolution of the system. The actual drawing of a curve using an iterative process such as Euler's method leads to difficulties associated with the iteration of rational functions. Figure 1a of [1] shows the behavior of Euler's method with step size $h=1$ for $f(z)=z^{3}-1$. In that figure, any initial point in the blue region will generate approximations which converge to the equilibrium solution at +1 . Euler's method with stepsize $h>0$ for (1.3) is

$$
z_{n+1}=z_{n}+h R\left(z_{n}\right)=z_{n}-h \frac{f\left(z_{n}\right)}{f^{\prime}\left(z_{n}\right)},
$$

the modified discrete Newton method of $f$, which is the ordinary discrete Newton method of $f^{1 / h}$. In particular, an equilibrium solution of (1.3) which is a multiple zero of $R$, and thus an essential singularity of $f$, is a parabolic fixed point of $z+h R(z)$, and is in the Julia set of $z+h R(z)$. Thus we consider a noniterative method for plotting trajectories which also allows the display of information about the time parametrization.

The trajectories of (1.1) and (1.4) are mutually orthogonal. If $R$ is the continuous Newton map of $f$, then $i R$ is the continuous Newton map of

$$
g(z)=f^{-i}(z) .
$$

Our considerations are based on the implicit solutions of (1.1), (1.4):

$$
\begin{aligned}
& f(z(t))=f(z(0)) e^{-t}, \\
& g(\zeta(t))=g(\zeta(0)) e^{-t} .
\end{aligned}
$$

From (6.3) and the precise definition of a solution given in Definition 2.11, we see that the ray in the $w$-plane containing $f(z(0))$ also contains $f(z(t))$, so that a procedure which plots a point $z$ if $f(z)$ is on the ray containing $f(z(0))$ would give the trajectory through $z(0)$. From the example $f(z)=z^{2}-1$, we see that if $f$ is many-to-one, then more than one trajectory will be plotted. This feature is an asset when it comes to plotting critical trajectories. 
If $f$ is multivalued, the problem arises that a computer works with only one branch of $f$, making it necessary to take into account the location of branch cuts, to assure that trajectories can be continued across them. The example $f(z)=z^{1+i}$, where only the principal value of $\arg z$ is used, illustrates this.

To characterize a given ray, we note that

$$
\log |g(z(t))|=\operatorname{Arg} f(z) \text {. }
$$

Thus $\log |g(z)|$ is constant on trajectories. Further, $\log |f(z)|$ is linear in time on the trajectories of (1.1). Thus plotting the level curves of $\log |f(z)|$ for equally spaced levels yields orthogonal trajectories defining bands of equal time width. To plot such curves, we consider the general problem of plotting the zero set of a real valued function $h(x, y)$.

Let $h(x, y)$ be a function of class $C^{1}$ on a rectangle $R$ defined by $x_{\min } \leq$ $x \leq x_{\max }, y_{\min } \leq y \leq y_{\max }$, and let $W$ be a rectangular window on a computer screen consisting of $N$ columns and $M$ rows of pixels, labeled 0 to $N-1$ and 0 to $M-1$ respectively. Let

$$
\begin{array}{cl}
\varepsilon_{x}=\left(x_{\max }-x_{\min }\right) / N, & \varepsilon_{y}=\left(y_{\max }-y_{\min }\right) / M, \\
x_{k}=x_{\min }+k \varepsilon_{x}, & y_{j}=y_{\min }+j \varepsilon_{y},
\end{array}
$$

and let $z_{j k}$ denote the center of the $j k$ rectangle:

$$
z_{j k}=x_{k}+i y_{j}+\frac{1}{2}\left(\varepsilon_{x}+i \varepsilon_{y}\right) .
$$

Thus the $j k$ th pixel represents a subrectangle $R_{j k}$ in $R$. Clearly $h$ can have a zero in $R_{j k}$ even if $h\left(z_{j k}\right) \neq 0$. Thus an algorithm which turns on pixel $j k$ if $h\left(z_{j k}\right)=0$ will fail to turn on all pixels containing a zero. On the other hand, a condition such as $\left|h\left(z_{j k}\right)\right| \leq \varepsilon$ for some $\varepsilon>0$ may turn on too many pixels and imbed the level set of $h$ in a broad strip. Thus a strategy is needed which is parsimonious with respect to computer resources, yet gives an informative picture of the zero set of $h$.

Let

$$
\delta=\frac{1}{2} \sqrt{\varepsilon_{x}^{2}+\varepsilon_{y}^{2}}
$$

and assume

$$
1 / 2 \sqrt{2}<\varepsilon_{2} / \varepsilon_{1}<2 \sqrt{2} .
$$

The pixel corresponding to $R_{j k}$ is turned on if

$$
h^{2}\left(z_{j k}\right)-\delta^{2}\left|\nabla h\left(z_{j k}\right)\right|^{2} \leq 0 .
$$

With this procedure, if $h$ is linear, then each pixel intersecting the zero set of $h$ will be activated, and the set of activated pixels will be at most two pixels wide.

To arrive at (6.8), we test for a zero of $h$ in $R_{j k}$ by comparing the signs of $h$ at the two points a distance $\delta$ from $z_{j k}$, in the direction of $\nabla h\left(z_{j k}\right)$. Thus the pixel corresponding to $r_{j k}$ is activated if

$$
h\left(z_{j k}+\delta e\right) h\left(z_{j k}-\delta e\right) \leq 0,
$$

where $e=\nabla h /|\nabla h|$ at $z_{j k}$. Linearization of $h$ in (6.9) yields (6.8). 
In the case that

$$
h(x, y)=\log |g(z)|-\log \left|g\left(z_{0}\right)\right|,
$$

where $z_{0}$ is a given initial point, then

$$
|\nabla h(x, y)|=\left|g^{\prime}(z) / g(z)\right|,
$$

so a pixel $z=z_{j k}$ is plotted if

$$
|g(z)|^{2}|h(x, y)|^{2}-\delta^{2}\left|g^{\prime}(z)\right|^{2} \leq 0 .
$$

To take into account the multivalued nature of $f$, rather than use $g=f^{-i}$, we use the explicit representation of $f$ given in (2.4), and let

$$
g(z)=e^{-i P_{0}(z)} \prod_{j=1}^{J}\left(z-z_{j}\right)^{-i A_{j}} e^{-i P_{j}\left(1 /\left(z-z_{j}\right)\right)} .
$$

Using (6.13), $\log |g(z)|$ is not restricted to the principal value of $\arg f(z)$. In the color plates, the white curves are pixels which are mapped onto the critical rays of $f$. Since $f$ is in general many-to-one, there can be noncritical trajectories which are mapped onto the critical ray. The color bands represent trajectories and orthogonal trajectories, and a particle moving according to (1.1) requires a fixed time to cross each orthogonal band.

\section{REFERENCES}

1. H. E. Benzinger, S. A. Burns, and J. I. Palmore, Chaotic complex dynamics and Newton's method, Phys. Lett. A 119 (1987), 441-446.

2. E. A. Coddington and N. Levinson, Theory of ordinary differential equations, McGraw-Hill, New York, 1955.

3. J. Ecalle, J. Martinet, R. Moussu, and J.-P. Ramis, Non-accumulation des cycles-limites. (I), C. R. Acad. Sci. Paris Sér. I 304 (1987), 375-377.

4. __, Non-accumulation des cycles-limites. (II), C. R. Acad. Sci. Paris Sér. I 304 (1987), 431-434.

5. M. W. Hirsch and S. Smale, On algorithms for solving $f(x)=0$, Comm. Pure Appl. Math. 32 (1979), 281-312.

6. Yu. S. II' yashenko, Theorems on the finiteness of limit cycles, Uspekhi Mat. Nauk 42 (1987), no. $3,223$.

7. L. M. Perko, On the accumulation of limit cycles, Proc. Amer. Math. Soc. 99 (1987), 515526.

8. H. Poincaré, Memoire sur les courbes définies par une equation différentielles, J. Mathematiques 7 (1881), 375-422; Ouevres (1880-1890), Gauthier-Villars, Paris, pp. 1-221.

9. Shi Songling, A concrete example of the existence of four limit cycles for plane quadratic systems, Sci. Sinica Ser. A 23 (1980), 153-158.

10. M. Shub, D. Tischler, and B. Williams, The Newton graph of a complex polynomial, SIAM J. Math. Anal. (to appear).

11. S. Smale, A convergent process of price adjustment and global Newton methods, J. Math. Econom. 3 (1976), 107-120. 
12. _ The fundamental theorem of algebra and complexity theory, Bull. Amer. Math. Soc. (N.S.) 4 (1981), 1-36.

13. $\ldots$, On the efficiency of algorithms of analysis, Bull. Amer. Math. Soc. (N.S.) 13 (1985), $87-121$.

Department of Mathematics, University of Illinois, 1409 W. Green Street, Urbana, ILLINOIS 61801 